\title{
UMA NOVA POSSIBILIDADE DE CONSTRUÇÃO DO CONHECIMENTO EM PSICOLOGIA
}

\author{
Leonardo Santos de Souza \\ Universidade do Oeste Paulista- UNOESTE \\ Cinthia de Sousa Noguchi \\ Universidade do Oeste Paulista- UNOESTE \\ Lucas Bondezan Alvares \\ Universidade do Oeste Paulista- UNOESTE
}

\begin{abstract}
Resumo
O número de ligas acadêmicas, definidas como organizações estudantis sem fins lucrativos, vêm crescendo no Brasil. No entanto, têm operado muitas vezes de modo incoerente com os objetivos preconizados pelo tripé universitário: ensino, pesquisa e extensão, sendo motivo de críticas constantes quando implementadas sem um processo crítico-reflexivo. O presente relato visa apresentar a experiência da Liga Acadêmica de Psicologia da Saúde (LAPS) em uma universidade no interior do Estado de São Paulo, destacando as práticas realizadas, os desafios e relevância deste novo recurso na formação do futuro psicólogo. Foi possível identificar que a LAPS contribuiu para a população local por meio da educação em saúde e que seus os desafios estão especialmente voltados à pesquisa. Nesse sentido, são necessários investimentos em produção científica e nos órgãos de representatividade das ligas universitárias, uma vez que as mesmas estão se apresentando como importantes ferramentas para a qualificação do futuro profissional.
\end{abstract}

Palavras-chave: ligas acadêmicas; psicologia da saúde; educação em saúde.

\section{A NEW POSSIBILITY OF CONSTRUCTION OF KNOWLEDGE IN PSYCHOLOGY}

\begin{abstract}
The number of academic leagues, defined as nonprofit student organizations, has been growing in Brazil. Nevertheless, sometimes they have worked in an incompatible way with the objectives advocated by university tripod: teaching, research and extension, being reason for critics when implemented without the critical-reflexive process. This report aims to present the experience of the Academic League of Health Psychology (ALHP) in a university of the interior of the state of São Paulo, emphasizing the practices, the challenges and relevance of this resource in the formation of the future psychologist. It was identified that the ALHP contributed to the local population through health education and that its challenges are especially tied to research. Therefore, it is necessary to invest in scientific production and in representatives' organs of university leagues, since they are presenting themselves as important tools for the qualification of the future professional.
\end{abstract}

Keywords: academic leagues; health psychology; health education. 


\title{
UNA NUEVA POSIBILIDAD DE CONSTRUCCIÓN DEL CONOCIMIENTO EN PSICOLOGÍA
}

\begin{abstract}
Resumen
El número de ligas académicas, definidas como organizaciones estudiantiles sin fines de lucro, vienen creciendo en Brasil. Sin embargo, han operado muchas veces de modo incoherente con los objetivos preconizados por el trípode universitario: enseñanza, investigación y extensión, de modo a recibir críticas constantes cuando implementadas sin un proceso crítico-reflexivo. El presente relato presenta la experiencia de la Liga Académica de Psicología de la Salud (LAPS) en una universidad al interior del Estado de São Paulo, destacando las prácticas realizadas, los desafíos y relevancia de este nuevo recurso en la formación de un psicólogo. Fue posible identificar que LAPS contribuyó para la población local por medio de la educación en salud y que sus desafíos están especialmente inclinados a la investigación. En este sentido, son necesarios investimentos en producción científica y en los órganos de representatividad de las ligas universitarias, una vez que las mismas están presentes como importantes herramientas para la calificación del futuro profesional.
\end{abstract}

Palabras clave: ligas académicas; psicología de la salud; educación en salud.

\section{INTRODUÇÃO}

A psicologia é uma ciência que permaneceu por muito tempo voltada aos aspectos clínicos e educacionais envolvidos no comportamento humano. Entretanto, à medida que surgiram novas demandas provocadas pelas configurações socioambientais, viu-se a necessidade de ampliar seus campos e limites de atuação. Um exemplo foi o campo da saúde que, a partir de seus avanços, colocou a Psicologia como uma importante referência de estudos e atuação (Angerami-Camon, 2000). A visão e os conceitos relacionados à definição de saúde também foram atualizados ao longo do tempo, deslocando-se do modelo biomédico e adotando o modelo biopsicossocial, já que o paradigma biomédico era insuficiente para a compreensão sobre o adoecer, por não considerar os fatores psicossociais e histórico-políticos do sujeito (DeMarco, 2006; Puttini, Pereira, \& Oliveira, 2010; Scliar, 2007; Silva, Viana, \& Paulino, 2011; Straub, 2014).

A concepção atual de saúde é definida pela Organização Mundial da Saúde (OMS) como o bem-estar biopsicossocial do sujeito, sem reduzi-la à ausência de doenças (World Health Organization, 1989/1948). Sendo assim, refere-se às práticas que consideram os aspectos físicos, orgânicos, espirituais, psicológicos, sociais, familiares e históricos, promovendo uma visão ampla e integral da pessoa adoecida, possibilitando que a equipe interdisciplinar realize as intervenções mais adequadas e uniformes em cada caso (Angerami-Camon, 2000; Silva et al., 2011; Straub, 2014).

Portanto, quando inserida em uma equipe interdisciplinar, a Psicologia da Saúde visa compreender a integração entre os fatores biopsicossociais que podem possuir caráter protetivo ou de vulnerabilidade à saúde do indivíduo. Possui a finalidade de promover, recuperar, manter a saúde e, ainda, prevenir novas ocorrências de adoecimento ou complicações através de estratégias que tornem o paciente autônomo e corresponsável pelo seu processo de saúde e 
doença (Angerami-Camon, 2000; Castro \& Bornholdt, 2004; Straub, 2014). A Psicologia da Saúde contempla desde os níveis primários aos terciários de atenção à saúde (Castro \& Bornholdt, 2004), podendo também se pensar, diante dos avanços técnicos-científicos, em um nível quaternário (Gorayeb, 2010), englobando centros para tratamentos de doenças específicas e/ ou as Unidades de Terapia Intensiva, indo ao encontro do que fora postulado por AngeramiCamon (2000). O psicólogo também tem como papel trabalhar contra a despersonalização da pessoa adoecida, ocasionada tanto pelo curso natural da doença, quanto pelo processo de institucionalização ao qual é acometida (Angerami-Camon, 2006).

Face ao exposto, é necessário pensar em uma formação em Psicologia aplicada a saúde que apresente ao aluno situações teóricas e práticas de forma interdisciplinar, para que o mesmo se torne agente de mudanças no cenário atual da saúde pública (Angerami-Camon, 2000; Hamamoto Filho, 2011; Magalhães, Rechtman, \& Barreto, 2015). Neste contexto, as ligas acadêmicas, definidas como grupos constituídos por docentes e alunos, em que os últimos perpassam por atividades de ensino, pesquisa e extensão universitária por meio de metodologias tradicionais e/ou ativas de ensino vêm sendo compreendidas como uma possibilidade formativa que vai de encontro à necessidade de um profissional dinâmico, justificando o crescimento desta modalidade de construção do conhecimento nos últimos anos (Hamamoto Filho, 2011; Magalhães et al., 2015).

\section{Histórico, conceituações e críticas às ligas acadêmicas}

Houve um grande crescimento na última década em relação à quantidade de ligas acadêmicas implementadas, especialmente nos cursos de Medicina (Hamamoto Filho, 2011). Uma liga acadêmica trata-se de uma organização estudantil ou entidade que não apresenta fins lucrativos, constituída por alunos de diferentes anos de graduação e administrada pelos mesmos, sob supervisão de profissionais/professores vinculados a Instituição de Ensino Superior. Nesse sentido, os membros participam de atividades que perpassam pelo tripé universitário (ensino, pesquisa, extensão), permitindo-os adquirir conhecimentos adicionais e serem atuantes em áreas específicas (Hamamoto Filho, 2011; Monteiro, Cunha, Oliveira, Bandeira, \& Menezes, 2008; Santana, 2012).

Consoante ao exposto pela literatura, Bastos, Trajman, Teixeira, Selig e Belo (2012), assim como Hamamoto Filho (2011), denotam que as atividades são implementadas de acordo com a gestão de cada liga, as quais variam entre aulas teóricas, práticas, atividades extensivas, simulações, eventos, entre outros. Dessa forma, as ligas fazem com que as vivências proporcionadas por suas práticas possam contribuir na escolha da futura especialidade dos alunos.

A primeira liga acadêmica brasileira foi criada em 1920 na Faculdade de Medicina da USP - São Paulo, sendo denominada como Liga de Combate à Sífilis. 
Esta liga surgiu a partir da identificação dos alunos sobre a necessidade de estratégias que possibilitassem intervenções contra a doença, que na época se proliferava nos grandes centros urbanos, especialmente em São Paulo, e a partir de então o número de ligas começou a se estender no Brasil (Burjato Júnior, 1999).

No entanto, o crescimento do número de ligas acadêmicas deve ser analisado com cautela, pois se as atividades de uma liga criam um currículo paralelo que supre uma formação universitária deficiente, corre-se o risco de uma especialização precoce. Tais fatores e diversos outros contingentes explicam uma série de críticas sofridas pelas ligas acadêmicas que, de fato, quando apresentam tais fragilidades, são compreensíveis (Hamamoto Filho, 2011; Monteiro et al., 2008). Além disso, é preciso estar atento ao conteúdo teórico e prático oferecido para que ela envolva todos os níveis do tripé universitário, variando os recursos pedagógicos utilizados, verificando sua eficácia e eficiência, bem como a motivação para sua criação, de projetos semelhantes e dos alunos que pretendem ingressar em uma liga acadêmica (Hamamoto Filho, 2011).

Como exposto pela literatura supracitada, frequentemente as ligas acadêmicas são mais presentes nos contextos médico-hospitalares. Porém, ao discutir conceitos como interdisciplinaridade e humanização no atendimento, fazse necessário que as demais áreas do saber, dentre elas inclusa a ciência psicológica, caminhem no mesmo ritmo, criando espaços para a formação de um pensamento mais crítico pelos graduandos acerca de temáticas que envolvem seu processo de formação e futura atuação profissional. Portanto, aqui se ressalta a necessidade apontada por Angerami-Camon (2000) de se pensar numa prática psicológica mais ampla, para compreensão do sujeito e seu comportamento frente ao processo de saúde e doença, condizente com os pressupostos que norteiam a Liga Acadêmica de Psicologia da Saúde (LAPS).

\section{Ligas acadêmicas e psicologia}

De acordo com Magalhães et al. (2015), as ligas acadêmicas dentro dos cursos de Psicologia são muito escassas, acontecimentos que corroboram tal afirmativa referem-se ao fato de que os únicos artigos que retrataram ligas acadêmicas de Psicologia foram os de Marques e Vieira (2012), que relataram uma experiência extensiva no município de Sobral (CE) e o artigo supracitado. Com o objetivo de realizar um mapeamento a nível brasileiro, Magalhães et al. (2015) desenvolveram um estudo transversal quantitativo utilizando descritores "Ligas acadêmicas" e "Psicologia", visando encontrar blogs e páginas que remetessem a ligas acadêmicas de Psicologia. Foram encontradas onze ligas, das quais cinco abordavam Análise do Comportamento, uma sobre Psicologia geral e as demais também se dividiam em unidades, nas seguintes especialidades: Saúde Mental, Psicanálise, Psicologia da Saúde, Psicologia Escolar e Psicologia Social. Em relação à localização geográfica, existe uma prevalência na região 
sudeste e não fora identificada diferença expressiva entre o número de ligas de Psicologia em universidades públicas e privadas. Isso indica que mesmo em um número pequeno, se comparada às ligas de Medicina, existe uma demanda, visto que a Psicologia brasileira tem começado a se apropriar deste recurso (Magalhães et al., 2015).

O presente artigo tem como objetivo principal relatar a experiência da criação da Liga Acadêmica de Psicologia da Saúde (LAPS) em uma universidade situada no oeste do estado de São Paulo. Além disso, busca-se destacar as práticas realizadas e temáticas abordadas na LAPS, bem como realizar uma análise crítica, refletindo a partir de seus desafios e possibilidades, a relevância desse novo recurso para a formação do futuro psicólogo inserido em equipes multiprofissionais e interdisciplinares.

\section{MÉTODO}

Trata-se de um estudo qualitativo, descritivo, na modalidade relato de experiência, retratando a repercussão produzida pela implementação da Liga Acadêmica de Psicologia da Saúde (LAPS) em uma universidade privada do interior do estado de São Paulo. A descrição dos resultados foi organizada inicialmente por uma breve apresentação histórica e metodológica da LAPS, bem como suas práticas, sucedidas de uma explanação dos principais desafios e possibilidades envolvidos na execução do projeto.

\section{RESULTADOS}

\section{A Liga Acadêmica de Psicologia da Saúde (LAPS)}

A Liga Acadêmica de Psicologia da Saúde é a primeira liga universitária em 28 anos de existência do curso de Psicologia de uma universidade do interior do estado de São Paulo. Foi idealizada por um aluno da instituição em dezembro de 2014 e implementada em agosto do ano seguinte. O projeto foi elaborado em conjunto com duas docentes desta mesma universidade, com o apoio da coordenadora do curso de Psicologia.

A Liga Acadêmica de Psicologia da Saúde (LAPS) tem como objetivos: promover um espaço para o conhecimento teórico e prático mais aprofundado sobre Psicologia da Saúde, propiciando uma análise crítica das demandas da atuação profissional, articulando o ensino, a pesquisa e a extensão por meio de profissionais da área da saúde e metodologias ativas, além das tradicionais, instrumentalizando os alunos para atuarem em equipes multi e interdisciplinares, além de conhecer a realidade regional do sistema de saúde e possibilidades de inserção profissional.

Para a realização dos encontros, foram utilizadas aulas expositivas com as seguintes temáticas: Introdução aos aspectos teóricos e prático-profissional do 
Psicólogo nos contextos de saúde; Introdução à Psicologia da Saúde; Introdução à Psicologia Hospitalar; Farmacologia e Psicologia da Saúde em casos de saúde mental; Atendimento psicossocial em saúde mental e atendimento domiciliar; Atendimento Multiprofissional em Saúde do Idoso; Saúde do trabalhador: Interlocuções entre o estresse e doenças cardiovasculares; Psico-Oncologia e Cuidados Paliativos. Adesão ao tratamento e formas de abordagem ao paciente (encontro entre ligas de Psicologia da Saúde e Enfermagem em Cardiologia). Até o momento foram recebidos como palestrantes: Psicólogos, Enfermeiros, Farmacêuticos, Fisioterapeutas, Nutricionistas, Médicos, profissionais de programas de residência multiprofissional em Saúde do Idoso, Urgência e Trauma e Intensivismo.

Além das aulas expositivas, foram realizadas mesas redondas sobre: Diálogo interdisciplinar em depressão pós-parto; Cardio-Oncologia e o fim da vida; Aulas teórico-práticas em laboratório de habilidades sobre Suporte Básico de vida cardiovascular e Primeiros socorros, em todos os semestres e Atuação multiprofissional em transplante e captação de órgãos. Essas temáticas foram discutidas e pensadas em conjunto com outras ligas da área da saúde, como a de cardiologia e oncologia, a fim de promover um diálogo entre as práticas dos diferentes saberes inseridos nas equipes de saúde.

Outros recursos utilizados foram as metodologias ativas, através de práticas simuladas/cenários comportamentais e híbridos no laboratório de Habilidades e Simulação, baseados na metodologia de Ensino Baseado em Simulação [EBS] (Araújo \& Quilici, 2012; Brandão, Collares, \& Marin, 2014; Santos \& Sato, 2012). Tais encontros foram nomeados como: "Casa simulada: Atendimento domiciliar em casos de saúde mental"; "Primeiros socorros em locais não estruturados"; "Simulação de parada cardiorrespiratória no consultório psicológico"; "Morte no hospital e o papel da equipe frente a esse contexto", sempre seguidos de Debriefing, abordado por Sastrías (2012) que o define como - momento após a simulação onde se reflete e analisa as condutas, comportamento e desenvolvimento dos participantes no cenário e suas implicações na realidade. Bem como encontros baseados em Problem Based Learning [Aprendizagem baseada em problemas], denominados: Lidando com a morte no hospital: O que vivencia o psicólogo, a família e a equipe (Borges, Chachá, Quintana, Freitas, \& Rodrigues, 2014; Borochovicius \& Tortella, 2014; Mello, Alves, \& Lemos, 2014; Vignochi, Benetti, Machado, \& Manfroi, 2009).

Já em relação à extensão universitária, foram realizadas as seguintes atividades: Visita técnica ao Hospital do Câncer de Barretos; Viagem interligas e participação do Simpósio multiprofissional em Dor do Hospital Sírio Libanês e na Jornada de Psicologia da Saúde, Psicossomática e Psico-oncologia da Universidade de São Paulo (USP). No fim de 2016, foi realizado o 10 Simpósio Multiprofissional de Psicologia da Saúde, com apresentações de trabalhos, mesas redondas e minicursos. 
Ainda no que diz respeito às atividades de extensão universitária, a LAPS ministrou palestras sobre Saúde do Trabalhador, na Semana Interna de Prevenção de Acidentes no Trabalho (SIPAT) para os servidores municipais do interior do estado de São Paulo a convite do município, abordando temáticas relacionadas ao estresse, burnout e suas implicações na qualidade de vida. Outras ações foram voltadas a prevenção/manutenção da hipertensão arterial e comorbidades em parceria com a Liga de Enfermagem em Cardiologia e o Projeto Pressão na Medida, no qual a população era avaliada e/ou acompanhada e recebia orientações de cunho multiprofissional e interdisciplinar a respeito de sua saúde. Alguns encaminhamentos eram realizados quando necessários. Vale ressaltar que antes de cada ação extensiva, os alunos eram capacitados pelos docentes e monitores do projeto, supervisionados pelos mesmos durante a realização dos atendimentos à comunidade e igualmente auxiliados na elaboração do material psicoeducativo que seria utilizado e distribuído nas ações preventivas à população.

No que diz respeito à pesquisa, a LAPS trabalha essencialmente com pesquisa básica, através dos dados coletados nas ações extensivas realizadas em parceria com a Liga de Enfermagem em Cardiologia e o projeto Pressão na Medida. Os resumos de trabalhos são apresentados e publicados em anais de congressos relacionados à Cardiologia multiprofissional/interdisciplinar e extensão universitária.

Devido à qualidade do projeto e do trabalho realizado, a LAPS recebeu em 2015 a certificação de "Padrão ouro" pelo Programa de Incubação de Ligas da Fundação Estudar, que é uma organização que tem como objetivo criar oportunidades para potencializar jovens talentos através de projetos que levam informação, preparação e financiamento para educação de excelência para que possam transformar a realidade as quais estão inseridas. Além disso, em 2016 foi premiada no Encontro Nacional de Ensino, Pesquisa e Extensão com o $1^{\circ}$ lugar na categoria "melhor projeto extensivo", que avaliou a qualidade dos projetos de maior impacto social e formação cidadã.

Principais desafios e limites enfrentados com a implementação da LAPS

No que diz respeito à ênfase ensino, especialmente no início da LAPS, houve uma grande dificuldade em encontrar palestrantes que ministrassem aulas ou cursos sem pró-labore ou recebendo um valor simbólico, uma vez que a maioria das ligas não dispõe de recursos financeiros abundantes. Ainda assim, através dos coordenadores discentes foram promovidas parcerias com diversos docentes, internos e externos à universidade, através da apresentação da proposta da LAPS, sua missão, valores e objetivos. A partir de então, os docentes que ainda não estavam acostumados com essa modalidade de projeto, começaram a aderir e promovê-lo gradualmente. Outro ponto a ser ressaltado é que, mesmo criando uma liga multi/interdisciplinar e divulgando aos demais 
cursos da área da saúde, foi possível perceber a dificuldade de adesão de outros cursos além da Psicologia. Então, a alternativa buscada pela LAPS para sanar esse déficit foi realizar parcerias com outras ligas acadêmicas e projetos extensivos multidisciplinares da universidade.

Em relação à pesquisa, verificou-se que foi a ação mais difícil a ser implementada, pois mesmo motivando todos participantes da liga, fomentando a importância da pesquisa para a atuação profissional, apenas cerca de $20 \%$ dos participantes aderiram a alguma modalidade de pesquisa dentro da LAPS, seja projetos de iniciação científica ou produção de resumos e outros trabalhos para congressos. Informalmente, a justificativa da maioria era a falta de tempo, visto que trabalhavam no contraturno da faculdade e/ou já participavam de outros projetos. Ainda no sentido das limitações vivenciadas pelos autores com relação à implementação da liga enquanto movimento estudantil, não foi possível encontrar material científico dentro da Psicologia que pudesse oferecer uma estrutura/modelo consistente para implementar a LAPS, levando os coordenadores a recorrer então à Medicina e Enfermagem, que ofereceram modelos mais embasados de trabalho, bem como recursos pedagógicos, tais como a Simulação realística e Problem based learning que foram adaptados para a Psicologia para aplicação na LAPS.

No eixo da extensão universitária, inicialmente, assim como na questão do ensino, foi difícil encontrar parceiros e locais onde ações voltadas à população pudessem ser realizadas, mas após alguns meses de apresentação das propostas da LAPS, foi possível, especialmente por intermédio dos demais projetos extensivos, inserir a liga de Psicologia na comunidade, através de projetos que, em sua maioria, visavam à triagem, educação em saúde, identificação de comportamentos de risco a saúde, encaminhamentos a outros dispositivos e realização de pesquisas através dos dados coletados.

\section{DISCUSSÃO}

Uma das maiores críticas expressas por Hamamoto Filho (2011) é que a maioria das ligas trabalha apenas com o ensino através de aulas teóricas e fechadas em sua própria área do saber, fracassando no objetivo ideológico de sua criação, uma vez que deveriam fundamentar-se na tríade ensino, pesquisa e extensão universitária e no diálogo multidisciplinar. A própria Medicina, considerada pioneira na implementação de ligas acadêmicas, apresenta baixa produção científica, com poucas publicações de alto nível de impacto, algo que se estende às demais áreas da saúde. Pois, segundo Santana (2012) existem muitos relatos de experiências e poucas pesquisas de campo, além do fato de muitas ligas trabalharem apenas com uma ênfase do tripé (pesquisa, ensino e extensão), focando especialmente no ensino, como aponta a literatura (Hamamoto Filho, 2011; Neves et al., 2008; Silva \& Flores, 2015). Com isso, é 
possível constatar o motivo pelo qual as publicações, inclusive as ações extensivas, encontram-se escassas ou pouco embasadas cientificamente.

Focar em apenas uma ênfase do tripé, em detrimento das demais, ao invés de proporcionar uma formação mais íntegra ao discente, reforça as críticas de uma especialização precoce, além de privá-lo de uma formação mais generalista que o capacitaria a enfrentar a gama de possibilidades com as quais possivelmente iria se deparar em sua futura atuação profissional. Com isso, promove-se a possibilidade de futuros profissionais que reproduzam um modelo biomédico apontado por DeMarco (2006) e/ou tão desastroso quanto, focando apenas em aspectos psicossociais, promovendo psicologismos e retardando o processo de compreensão global do paciente e consequentemente seu prognóstico (Hamamoto Filho, 2011; Silva et al., 2011).

Para que se possa prevenir a ocorrência de tais problemáticas, é preciso avaliar a motivação do aluno que ingressa em uma liga para que não corresponda justamente a uma possível demanda de especialização precoce procurada pelo estudante, visto que um dos motivos pelos quais os alunos buscam projetos/ligas é a possibilidade de atividades práticas. Tais pontos são possíveis indicativos da distância entre conteúdos que a grade curricular da universidade oferece e as expectativas do aluno, ou até mesmo do que o aluno recebe da graduação e as exigências do mercado de trabalho. Dessa forma, as Instituições de Ensino Superior que operam por meio de uma grade curricular deficitária, correm o risco, se não analisado criticamente, de esperar que ligas acadêmicas supram essa demanda (Hamamoto Filho, 2011; Taquette, CostaMacedo, \& Alvarenga, 2003).

O estudo de Magalhães et al. (2015) apesar de produzir avanços no sentido de produção científica para ligas de Psicologia, apresenta vieses em seu método, uma vez que o critério de busca limitou-se a alguns termos em redes sociais, o que não oferece um resultado fidedigno. Um fator que contribui para essa problemática das ligas acadêmicas é não haver um órgão regulamentador ou conselho representativo que forneça dados mais objetivos quanto ao trabalho das ligas universitárias em geral, com exceção da medicina que possui a ABLAMAssociação Brasileira de Ligas Acadêmicas de Medicina. Acredita-se que existindo um órgão bem difundido, que regulamente e dê suporte às ligas acadêmicas multiprofissionais, os déficits diminuam, bem como venha facilitar o avanço científico na área, possibilitando um mapeamento real das ligas acadêmicas, especialmente na Psicologia.

Salientando novamente a questão do Ensino Baseado em Simulação, não foram encontrados trabalhos com o uso dessa ferramenta dentro dos contextos de formação em Psicologia, apesar da literatura, especialmente Santos \& Sato (2012), abordar o uso deste recurso pela e para a equipe multidisciplinar de saúde, diferente do uso já evidenciado da Aprendizagem Baseada em Problemas, visto em Kodjaoglanian et al. (2003). Entretanto, uma Psicologia que deseja 
avançar na produção e construção do conhecimento precisa estar disposta a adaptar-se constantemente às novas demandas, como proposto por AngeramiCamon (2000), formando seus discentes com maior qualidade através de metodologias ativas de ensino, minimizando as possibilidades de danos ao paciente usuário dos serviços de saúde, durante e posterior à graduação (Araújo \& Quilici, 2012; Brandão et al., 2014; Borges et al., 2014; Mello et al., 2014; Vignochi et al., 2009; ).

Os alunos que têm a oportunidade de simular atendimentos próximos aos reais e vivenciam experiências de atendimento em ambiente controlado saem mais preparados para o exercício profissional responsável, uma vez que essa metodologia permite fornecer feedback aos alunos sobre o que está adequado e o que precisa ser trabalhado para que ele forneça um serviço de qualidade, sem oferecer riscos ao paciente. Essa metodologia aumenta a segurança dos pacientes já que os alunos aprendem não só sobre a sua prática isolada, mas sobre as interfaces do contexto inter e multidisciplinar, simulando antes, para só então realizarem os atendimentos à população (Araújo \& Quilici, 2012; Brandão et al., 2014; Dourado \& Giannella 2014; Kaneko et al., 2015; Santos \& Sato, 2012).

Tal fato corrobora as proposições de Magalhães et al. (2015) no sentido de as ligas serem instrumentos importantes para os universitários, uma vez que promovem oportunidades de pesquisa e promoção de saúde à comunidade de maneira integrada, além de favorecer o protagonismo do graduando para se apropriar do conhecimento. Mesmo as questões de interdisciplinaridade sendo constantes e abordadas na comunidade científica há muito tempo, esbarra-se em velhos entraves para uma promoção de saúde igualitária, pois atua-se de forma multiprofissional, ao passo que a interdisciplinaridade fica em segundo plano, produzindo, assim, divergências entre teoria e prática (Angerami-Camon, 2000). Tal fato reflete na concepção das ligas e de como elas trabalham, vindo este a ser outro fator que possa explicar a ênfase em um ou outro pilar do tripé

universitário supracitado, impossibilitando uma atuação holística e descentralizadora na prática profissional quando chegarem ao mercado de trabalho (Hamamoto Filho, 2011; Neves et al., 2008; Silva \& Flores, 2015).

\section{CONSIDERAÇÕES FINAIS}

Face ao exposto, foi possível identificar que a Liga Acadêmica de Psicologia da Saúde vem tentando trabalhar em todas as ênfases do tripé, porém, em consonância com a literatura, de maneira insuficiente no que diz respeito à pesquisa. Entretanto, é nítida a relevância da LAPS na vida universitária de seus membros, compreendida por meio do reconhecimento acerca do impacto gerado pelo projeto, assim como dos feedbacks recebidos dos membros e da literatura por sua viabilidade de aplicação e importância para a atuação profissional. 
Alunos que participam de projetos extensivos neste modelo têm a oportunidade de aprimorar seu repertório teórico e prático promovendo melhor qualidade de vida à população local de forma segura. Consequentemente, chegam ao mercado de trabalho mais capacitados e preparados para as demandas complexas do sistema de saúde. A aproximação das ligas acadêmicas com profissionais e projetos de pesquisa/extensão de outras áreas potencializa ainda mais a construção de um conhecimento que vai de encontro com as atuais demandas do paciente no contexto de saúde. Espera-se que este trabalho possa servir de material para discentes e docentes que se interessem na implementação de ligas acadêmicas, especialmente dentro da Psicologia, produzindo novos saberes à comunidade científica e local, fomentando a criação e implementação de órgãos de representação que forneçam diretrizes e suporte às ligas universitárias.

\section{DECLARAÇÃO DE CONFLITOS DE INTERESSE}

Não há conflito de interesse.

\section{REFERÊNCIAS}

Angerami-Camon, V. A. (2000). Psicologia da saúde: Um novo significado para a prática clínica. São Paulo, SP: Pioneira.

Angerami-Camon, W. A. (2006). O psicólogo no hospital. In W. A. AngeramiCamon, F. A. R. Trucharte, R. B. Knijnik, \& R. W. Sebastiani (Eds.), Psicologia hospitalar: Teoria e prática (pp. 15-28). São Paulo, SP: Thomson Learning.

Araújo, A. L. L. S, \& Quilici, A. P. (2012). O que é simulação e por que simular. In A. P. Quilici, K. Abrão, S. Timerman, \& F. Gutierrez (Eds.), Simulação clínica: Do conceito à aplicabilidade (pp. 1-16). São Paulo, SP: Atheneu.

Bastos, M. L. S., Trajman, A., Teixeira, E G., Selig, L., \& Belo, M. T. C. T. (2012). O papel das ligas acadêmicas na formação profissional. Jornal Brasileiro de Pneumologia, 38(6), 803-805. http://dx.doi.org/10.1590/S180637132012000600018.

Borges, M. C, Chachá, S. G. F., Quintana, S. M., de Freitas, L. C. C., \& Rodrigues, M. D. L. V. (2014). Aprendizado baseado em problemas. Medicina, 47(3), 301-307. http://dx.doi.org/10.11606/issn.21767262.v47i3p301-307.

Borochovicius, E., \& Tortella, J. C. B. (2014). Aprendizagem Baseada em Problemas: Um método de ensino-aprendizagem e suas práticas educativas. Ensaio: Avaliação e Políticas Públicas em Educação, 22(83), 263-294. http://dx.doi.org/10.1590/S0104-40362014000200002. 
Brandão, C. F., Collares, C. F., \& Marin, H. F. (2014). A simulação realística como ferramenta educacional para estudantes de medicina. Scientia Medica, 24(2), 187-192. Recuperado em 29 de abril de 2019, de http://revistaseletronicas.pucrs.br/ojs/index.php/scientiamedica/article/vie w/16189/11485.

Burjato Júnior, D. (1999). História da liga de combate à sífilis e a evolução da sífilis na cidade de São Paulo (1920-1995). (Dissertação de Mestrado). Universidade de São Paulo, São Paulo.

Castro, E. K., \& Bornholdt, E. (2004). Psicologia da saúde x psicologia hospitalar: Definições e possibilidades de inserção profissional. Psicologia: Ciência e Profissão, 24(3), 48-57. http://dx.doi.org/10.1590/S141498932004000300007.

DeMarco, M. A. (2006). Do modelo biomédico ao modelo biopsicossocial: Um projeto de educação permanente. Revista Brasileira de Educação Médica, 30(1), 60-72. http://dx.doi.org/10.1590/S0100-55022006000100010.

Dourado, A. S. S., \& Giannella, T. R. (2014). Ensino baseado em simulação na formação continuada de médicos: Análise das percepções de alunos e professores de um Hospital do Rio de Janeiro. Revista Brasileira de Educação Médica, 38(4), 460-469. http://dx.doi.org/10.1590/S010055022014000400007.

Gorayeb, R. (2010). Psicologia da saúde no Brasil. Psicologia: Teoria e Pesquisa, 26(especial), 115-122. http://dx.doi.org/10.1590/S010237722010000500010.

Hamamoto Filho, P. T. (2011). Ligas Acadêmicas: Motivações e críticas a propósito de um repensar necessário. Revista Brasileira de Educação Médica, 35(4), 535-543. http://dx.doi.org/10.1590/S010055022011000400013.

Hamamoto Filho, P. T., Villas-Bôas, P. J. F., Corrêa, F. G., Muñoz, G. O. C., Zaba, M., Venditti, V. C., ... Schellini, S. A. (2010). Normatização da abertura de ligas acadêmicas: A experiência da Faculdade de Medicina de Botucatu. Revista Brasileira de Educação Médica, 34(1), 160-167. http://dx.doi.org/10.1590/S0100-55022010000100019.

Kaneko, R. M. U., Couto, T. B., Coelho, M. M., Taneno, A. K., Barduzzi, N. N., Barreto, J. K. S., ... Carvalho, F. S. (2015). Simulação in Situ, uma metodologia de treinamento multidisciplinar para identificar oportunidades de melhoria na segurança do paciente em uma unidade de alto risco. Revista Brasileira de Educação Médica, 39(2), 286-293. http://dx.doi.org/10.1590/1981-52712015v39n2e00242014. 
Kodjaoglanian, V. L., Benites, C. C. A., Macário, I., Lacoski, M. C. E. K., Andrade, S. M. O., Nascimento, V. N. A., ... Machado, J. L. (2003). Inovando métodos de ensino-aprendizagem na formação do psicólogo. Psicologia: Ciência e Profissão, 23(1), 2-11. http://dx.doi.org/10.1590/S141498932003000100002.

Magalhães, E. P., Rechtman, R., \& Barreto, V. (2015). A liga acadêmica como ferramenta da formação em Psicologia: Experiência da LAPES. Psicologia Escolar e Educacional, 19(1), 135-141. http://dx.doi.org/10.1590/21753539/2015/0191813.

Marques, G. H., \& Vieira, V. C. A. L (2012). Liga de psicologia hospitalar: Uma experiência na promoção de saúde no município de Sobral. Scientia, 1(1), 1-8. Recuperado em 29 de abril de 2019, de http://www.faculdade.flucianofeijao.com.br/site novo/scientia/servico/pdfs/ psicologia/Revista Scientia PSI 03.pdf.

Mello, C. C. B., Alves, R. O., \& Lemos, S. M. A. (2014). Metodologias de ensino e formação na área da saúde: Revisão de literatura. Revista CEFAC, 16(6), 2015-2028. http://dx.doi.org/10.1590/1982-0216201416012.

Monteiro, L., Cunha, M., Oliveira, W., Bandeira, N., \& Menezes, J. (2008). Ligas acadêmicas: O que há de positivo? Experiência de implantação da Liga Baiana de Cirurgia Plástica. Revista Brasileira de Cirurgia Plástica, 23(3), 158-161. Recuperado em 29 de abril de 2019, de http://www.rbcp.org.br/export-pdf/405/23-03-05.pdf.

Neves, F. B. C. S., Vieira, P. S., Cravo, E. A., Dias, M., Bitencourt, A., Guimarães, H. P., ... Orlando, J. M. C. (2008). Inquérito nacional sobre as ligas acadêmicas de medicina intensiva. Revista Brasileira de Terapia Intensiva, 20(1), 43-48. http://dx.doi.org/10.1590/S0103507X2008000100007.

Puttini, R. F., Pereira, A. Jr., \& Oliveira, L. R. (2010). Modelos explicativos em saúde coletiva: Abordagem biopsicossocial e auto-organização. Physis: Revista Saúde Coletiva, 20(3), 753-767. http://dx.doi.org/10.1590/S0103-73312010000300004.

Santana, A. C. D. A. (2012). Ligas acadêmicas estudantis: O mérito e a realidade. Medicina, 45(1), 96-8. Recuperado em 29 de abril de 2019, de http://revista.fmrp.usp.br/2012/vol45n1/PV Ligas\%20Acad\%EAmicas\%20E studantis.pdf.

Santos, E. C., \& Sato, S. N. (2012). Simulação na graduação de profissionais da saúde. In A. P. Quilici, K. Abrão, S. Timerman, \& F. Gutierrez (Eds.). Simulação clínica: Do conceito à aplicabilidade (pp. 27-47). São Paulo, SP: Atheneu.

Sastrías, J. M. F. (2012). Debriefing. In A. P. Quilici, K. Abrão, S. Timerman, \& F. Gutierrez (Eds.). Simulação clínica: Do conceito à aplicabilidade (pp. 8792). São Paulo, SP: Atheneu. 
Scliar, M. (2007). História do conceito de saúde. Physis: Revista de Saúde Coletiva, 17(1), 29-41. http://dx.doi.org/10.1590/S010373312007000100003.

Silva, S. A., \& Flores, O. (2015). Ligas acadêmicas no processo de formação dos estudantes. Revista Brasileira de Educação Médica, 39(3), 410-417. http://dx.doi.org/10.1590/1981-52712015v39n3e02592013.

Silva, K. A., Viana, H.A., \& Paulino, L. R. S. R. (2011). Perspectivas, reflexões e desafios dos modelos biomédico e biopsicossocial em psicologia. Actas do $16^{a}$ Encontro Nacional da ABRAPSO, Recife, PE. Recuperado em 29 de abril e 2019,

de

http://www.encontro2011.abrapso.org.br/trabalho/view?q=YToyOntzOjY6In BhcmFtcyI7czozNjoiYToxOntzOjExOiJJRF9UUkFCQUxITyI7czo0OiIyMzczIjt9Ij tzOjE6ImgiO3M6MzI6ImRjZTYyODBhYmFIZjI5MWEwNmNiMTk4YTNmZjAzM WQXIjt9

Straub, R. O. (2014). Psicologia da saúde: Uma abordagem biopsicossocial. Porto Alegre, RS: Artmed Editora.

Taquette, S. R., Costa-Macedo, L. M. D., \& Alvarenga, F. D. B. (2003). Currículo paralelo: Uma realidade na formação dos estudantes de medicina da UERJ. Revista Brasileira Educação Médica, 27(3), 171-176.

Vignochi, C. M., Benetti, C. D. S., Machado, C. L. B., \& Manfroi, W. C. (2009). Considerações sobre aprendizagem baseada em problemas na educação em saúde. Revista HCPA. Porto Alegre. 29(1), 45-50. Recuperado em 29 de abril

de 2019,

de

https://www.lume.ufrgs.br/bitstream/handle/10183/157866/000829624.pd f?sequence $=1$ \&isAllowed $=\mathrm{y}$.

World Health Organization. (1989). Constitution of the world health organization (37 ed.). Geneva: World Health Organization. (Original publicado em 1948).

Sobre os autores

Leonardo Santos de Souza é psicólogo pela Faculdade de Ciências da Saúde da Universidade do Oeste Paulista (UNOESTE). Especialista em Docência e Gestão na Educação Superior pela mesma Universidade e Mestrando pelo Programa de Pós-graduação Stricto Sensu em Educação (Bolsista CAPES) da UNOESTE. Pós graduando em Psicologia da Saúde e Hospitalar pela Faculdade de Medicina de São José do Rio Preto (FAMERP). Fundador e Ex-Presidente da Liga Acadêmica de Psicologia da Saúde (LAPS)- UNOESTE. leonardosouza.psicologia@gmail.com Cinthia de Sousa Noguchi é psicóloga pela Faculdade de Ciências da Saúde da Universidade do Oeste Paulista (UNOESTE). Mestranda pelo Programa de PósGraduação Stricto Sensu em Educação (Bolsista CAPES) na mesma Universidade. Ex-Presidente da Liga Acadêmica de Psicologia da Saúde (LAPS)- UNOESTE. ctnoguchi2@gmail.com 
Lucas Bondezan Alvares é psicólogo pela Universidade do Oeste Paulista (UNOESTE). Especialista em Psicologia e Saúde pela Universidade Estadual Paulista Júlio de Mesquita Filho. Possui Mestrado Profissional em Educação das Profissões da Saúde pela PUC/SP. Professor na UNOESTE e atualmente coordenador docente da Liga Acadêmica de Psicologia da Saúde (LAPS)UNOESTE. lucas_psicologia@yahoo.com.br

A contribuição de cada autor pode ser atribuída como se segue: L.S.S e C.S.N contribuíram para a conceitualização do estudo, organização dos resultados e visualização do artigo; L.S.S. fez a redação inicial do artigo (rascunho) e L.S.S, C.S.N e L.B.A são os responsáveis pela redação final do manuscrito (revisão e edição).

Os autores agradecem à Caroline da Cruz Pavan-Cândido e Regina Gioconda de Andrade pelo apoio nas fases iniciais da LAPS. Agradecem, ademais, Camélia Santina Murgo pela leitura da versão inicial do texto.

Recebido em: 01/06/2017

$1^{\text {a }}$ revisão em: 04/01/2018 Aceito em: 05/04/2018 